\title{
Regenerative Impact (Capacity) of Cerebrolysin versus N-Acetyl- Cysteine on Facial Nerve injury in Rats
}

\author{
Original Hani A. M. Aldheeb, Mai A. Haggag ${ }^{a}$, Rehab Rizk EL-Zehary, \\ Article \\ Nahed Ebraheem Ghoneim ${ }^{a}$ \\ ${ }^{a}$ Department of Oral and Maxillofacial Surgery, ${ }^{b}$ Department of Oral Biology, \\ Faculty of Dentistry, Mansoura University, Mansoura, Egypt
}

\begin{abstract}
Background: Facial nerve (FN) injury is an important health issue worldwide and the most essential principle for all surgeons is proper diagnosis and early treatment.

Aim of the study: Evaluation of the effect of Cerebrolysin (CBL) versus N-acetyl-cysteine (NAC) on FN regeneration. Materials and Methods: Normal 24 Sprague Dawely adult male rats were selected with a weight range 250 - 300 gm. Crushtype FN injury was performed on the right side of all rats then, they were randomly divided into three equal groups of eight rats each. In group I, the rats were left without any treatment and considered as a control. Rats of group II received $50 \mathrm{mg} /$ $\mathrm{kg}$ /day NAC intraperitoneally for a period of 28 days while rats of group III received $2.5 \mathrm{ml} / \mathrm{kg} /$ day CBL intraperitoneally for the same period. Postoperatively, eye-blink reflex was used to evaluate the functional recovery after the $2^{\text {nd }}$ and the 4 th weeks. All animals were euthanized by overdose of diethyl ether after 28 days and nerve regeneration was evaluated based on histopathological and immunohistochemical (IHC) examinations. All data collected were analyzed using SPSS version 20.0 software. Statistical significance was inferred at a $P$ value $\leq 0.05$.

Results: Nerve regeneration was obvious in group III with significant improvement of the eye-blink reflex at 2 weeks period that became non-significant at 4 weeks. Histological and IHC evaluation revealed high regeneration and minimum inflammation in group III when compared with groups I and II.

Conclusion: $\mathrm{CBL}$ enhances nerve regeneration and rehabilitation better than NAC after facial nerve crush injury in rats.
\end{abstract}

Key Words: Cerebrolysin, Crush injury, Facial nerve, Nerve regeneration, N-acetyl-cysteine.

Received: 12 March 2020, Accepted: 29 April 2020.

Corresponding Author: Mai Ahmed Haggag, Department of Oral and Maxillofacial Surgery, Faculty of Dentistry, Mansoura University, Mansoura , Egypt, Tel.: +20502258204, Mobile: +201224646605, E-mail: drmaihaggag5@gmail.com.

ISSN: 2090-097X, October 2020, Vol. 11, No. 4

\section{INTRODUCTION}

The facial nerve (FN), also known as, the 7th cranial nerve, is a mixed nerve with motor, sensory, and parasympathetic fibers. Its motor fibers control facial expression and loss of its function leads to many emotional and aesthetic problems. Its affected function may differ from slight disfigurement to total loss of function, according to the severity of trauma. FN has five major branches: temporal, zygomatic, buccal, marginal mandibular, and cervical $^{[1]}$.

The anatomical course of FN is very complex, and dysfunction can be due to traumatic, congenital, infectious, inflammatory, and - or neoplastic etiologies ${ }^{[2]}$. Additionally, most of the otological and otoneurological procedures, surgeries of the parotid gland and the face are performed around the FN. So that, it is in vulnerability to iatrogenic injuries $^{[3]}$.

Nerve injuries were classified into neuropraxia, axonotmesis and neurotmesis ${ }^{[4]}$. The assessment of patients with facial paralysis has three main purposes: to detect the site of the injury, the grade of dysfunction and the cause $^{[5]}$.

Management of FN injury in the present time focuses on that may or may not heal spontaneously ${ }^{[6]}$. The development in microsurgery, microbiology, radiographic imaging and electro-diagnostic testing has provided great vision into the treatment, diagnosis, pathophysiology and rehabilitation of FN. The proper diagnosis and early treatment is the most important principle for all physicians in management of FN pathology ${ }^{[5]}$.

However, the treatment with surgical intervention is essential in injuries associated with immediate complete paralysis, which are unlikely to have spontaneous recovery, because of the presumed neuronal disorder ${ }^{[6]}$.

Many therapeutic agents (such as: steroids, nimodipine, oxytocin...etc.) have been suggested for years to improve neural function and shorten recovery period, and investigations still go on ${ }^{[7,8]}$. 
$\mathrm{N}$-acetyl-cysteine is acetyl derivative of the amino acid cysteine ${ }^{[9]}$. Some of studies demonstrate the antiinflammatory, anti-apoptotic, and pro-regenerative effects of NAC treatment after spinal cord injury. These effects are detected at an early point after initial trauma and also persist over a period of several weeks postoperatively ${ }^{[10]}$.

Cerebrolysin is a porcine brain-derived peptide preparation that has pharmacodynamic properties similar to those of endogenous neurotrophic factors because it has neurotrophic effects ${ }^{[11]}$.

From the forementioned data it was of interest to compare the effect of CBL with that of NAC and also if these drugs have benefits over spontaneous healing in certain types of facial nerve injury.

\section{MATERIALS AND METHODS}

Twenty-four Sprague Dawely rats with average weight of (250---300 g), were lived under a good condition with free access to food and water. They were provided from Medical Experimental Research Center (MERC), Mansoura, Egypt. All experimental procedures were performed according to a protocol approved by the ethical committee of Faculty of Dentistry, Mansoura University, Egypt. Animals were exposed to a 12-hour day/night cycle, $22-25^{\circ} \mathrm{C}$ and stable air humidity of $65-70 \%$ was kept constant.

\section{Preoperative assessment:}

On the day of surgery, before animals were anesthetized, a saline drop was placed from a pipet onto the right eye to determine the presence of the blink reflex ${ }^{[12]}$.

\section{Surgical procedure:}

The animals were anesthetized by intraperitoneal injection of xylazine $(0.5 \mathrm{ml} / \mathrm{kg})$ and ketamine $(0.9 \mathrm{ml} / \mathrm{kg})^{[13]}$. Then, Skin on the facial nerve trace were shaved and cleaned by Betadine followed by $70 \%$ ethanol, and dried ${ }^{[14]}$. A pre-auricular incision with a marginal mandibular extension was made on the right side of the face to expose the trunk of facial nerve ${ }^{[15]}$. The trunk of FN was crushed for 1 minute via a hemostatic mosquito clamp to three clicks (Figure 1) ${ }^{[12]}$.

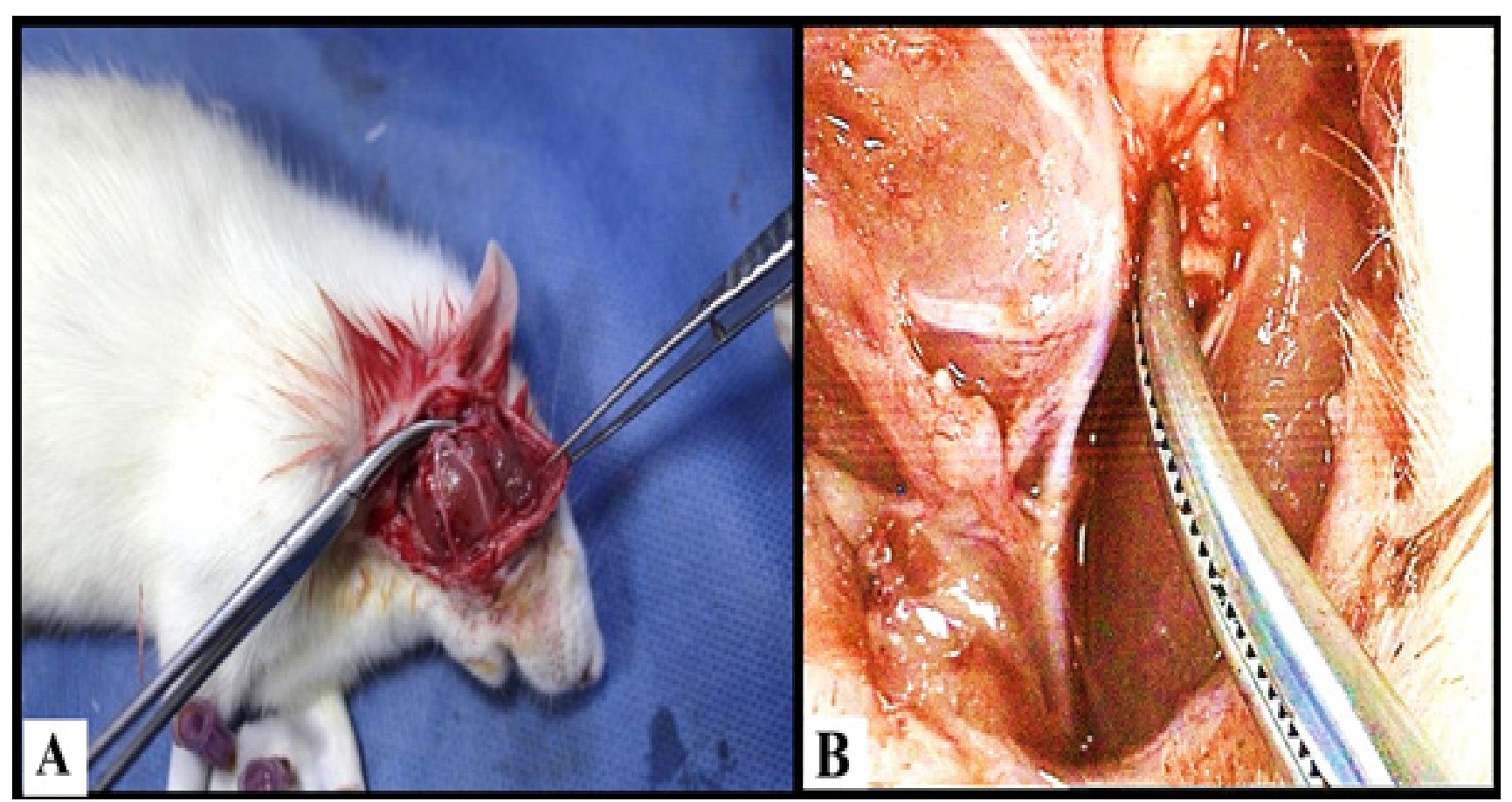

Figure 1: (A) Photographic picture showing FN trunk crushing by a standard micro-hemostat for 1 min. (B) microscopic picture showing FN trunk crushing. 


\section{Grouping of the animals:}

The rats were randomly and equally divided into three groups:

\section{Group I: (Control group):}

Included eight rats in which right FN trunk was crushed and left without treatment.

\section{Group II: (Study group I):}

Included eight rats which intraperitoneally injected with NAC at a dose of $50 \mathrm{mg} / \mathrm{kg} /$ day for a period of 28 days after nerve crushing ${ }^{[12]}$.

\section{Group III: (Study group II):}

Included eight rats which intraperitoneally injected with CBL at a dose of $2.5 \mathrm{ml} / \mathrm{kg} /$ day for a period of 28 days after nerve crushing ${ }^{[16]}$.

\section{Postoperative assessment:}

Functional testing was made at the day after operation. During 28 days the rats were tested at 2 and 4 weeks postoperatively ${ }^{[12]}$.

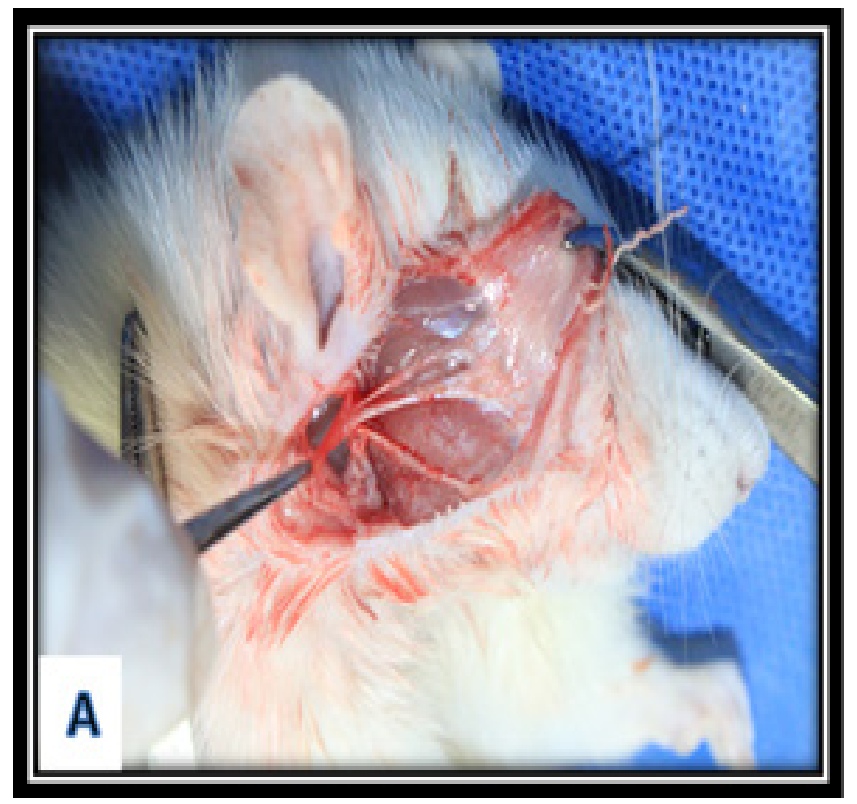

Scale of eye closing and blinking reflex observation ${ }^{[17]}$ :

\begin{tabular}{cc}
\hline Score & Movement \\
\hline $\mathbf{1}$ & No movement \\
$\mathbf{2}$ & Contraction $/$ no closure \\
$\mathbf{3}$ & $50 \%$ closure \\
$\mathbf{4}$ & $75 \%$ closure \\
$\mathbf{5}$ & Complete closure \\
\hline
\end{tabular}

\section{Histological analysis:}

After 28 days from the first day of nerve crushing, animals of each group were euthanized by overdose of diethyl ether and then decapitated. The specimens involving FN trunk and their branches were separated from muscles (Figure 2) for histological and IHC analysis and immediately placed in $10 \%$ buffered formalin solution for 48 hours, dehydrated in ascending grades of ethyl alcohol (70 - $100 \%)$, cleared in xylene, and embedded in paraffin. Five-micron thick paraffin sections were prepared using a microtome and slides prepared from the sections were stained with hematoxylin and eosin stain $(\mathrm{H}$ and $\mathrm{E})$ and immunohistochemically for Nuclear factor kappa-B (NF-kB) and S100 proteins then examined under microscope $\mathrm{e}^{[18]}$.

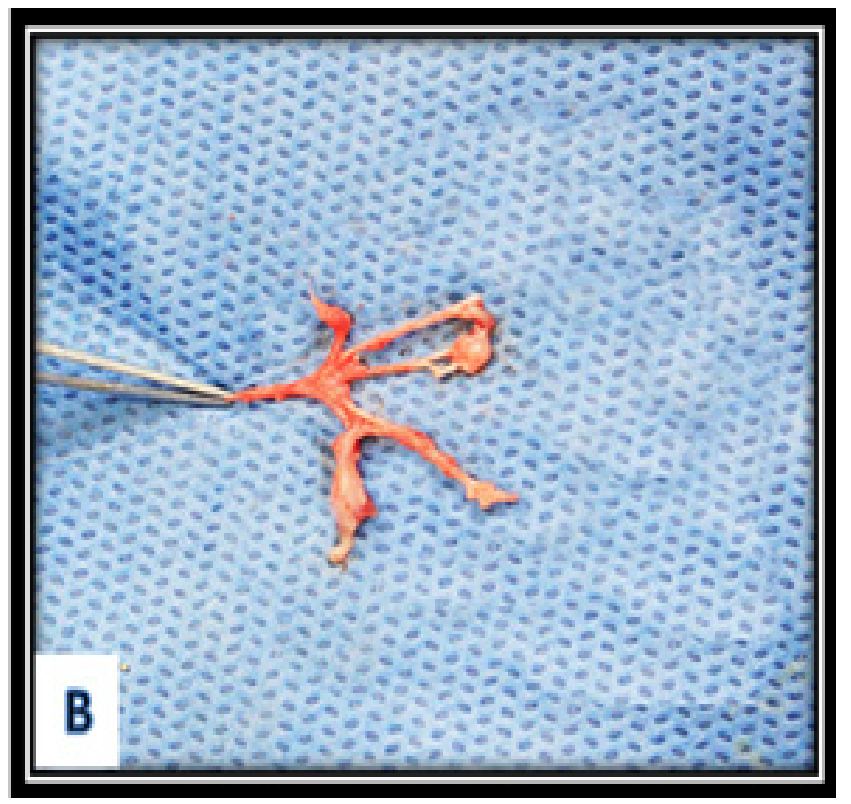

Figure 2: Showing: (A) Separation of FN trunk and their branches, (B) Final specimen. 


\section{Statistical Analysis:}

The data were put into a table, coded then analyzed using SPSS software package version 20.0 (IBM, computer program). Data were summarized as means and standard deviation. Mean value were compared by (ANOVA) One-way variation analysis test to compare between the groups. The level of significance was set at $\leq 0.05$ for all statistical analysis and confidence interval at $95 \%(95 \%$ CI). Pairwise comparison between each 2 groups was done using (Post Hoc) Test.

\section{RESULTS}

\section{A. Functional evaluation (eye blinking):}

The functional index score was determined on the basis of eye blink reflex via video analysis. Fig. 3 displays the progression of eye blinking over the studied time using a standardized scale for rat eye blink function.

Kruskal Wallis test for function (eye blinking) at two weeks' period in all groups representing statistically significant differences between the groups $(\boldsymbol{P}=\mathbf{0 . 0 0 7})$ while at four weeks' period there was no statistically significant difference between all study groups $(\boldsymbol{P}=\mathbf{0 . 2 5 8})$.

Post Hoc test for comparison between each 2 groups representing no statistically significant differences between control group and NAC group $\left(\boldsymbol{P}_{1}=\mathbf{0 . 5 0 6}\right)$, while statistically significant differences were reported between control group and CBL group $\left(\boldsymbol{P}_{2}=\mathbf{0 . 0 0 3}\right)$, and between NAC group and $\mathrm{CBL}$ group $\left(\boldsymbol{P}_{3}=\mathbf{0 . 0 2}\right)$ as shown in (Table 1 and Figure 3).

Table 1: Comparison between the three studied groups according to functional testing (eye reflex score):

\begin{tabular}{lccccc}
\hline $\begin{array}{c}\text { Functional } \\
\text { testing } \\
\text { (eye reflex } \\
\text { score) }\end{array}$ & $\begin{array}{c}\text { Control } \\
(\mathbf{n}=\mathbf{8})\end{array}$ & $\begin{array}{c}\text { N-Acetyl } \\
\text { cysteine } \\
(\mathbf{n}=\mathbf{8})\end{array}$ & $\begin{array}{c}\text { Cerebrolysin } \\
(\mathbf{n}=\mathbf{8})\end{array}$ & $\mathbf{H}$ & $\boldsymbol{P}$ \\
\hline $\mathbf{2}$ weeks & & & & & \\
Min. - Max. & $2.0-3.0$ & $2.0-4.0$ & $3.0-4.0$ & & \\
Mean \pm SD. & $2.50 \pm 0.53$ & $2.75 \pm 0.71$ & $3.63 \pm 0.52$ & $9.819^{*}$ & $0.007^{*}$ \\
Median & 2.50 & 3.0 & 4.0 & & \\
Sig. bet. & $P_{1}=0.506, P_{2}=0.003^{*}, P_{3}=0.02^{*}$ & & \\
groups & & & & & \\
4 weeks & $4.0-5.0$ & $4.0-5.0$ & $4.0-5.0$ & & \\
Min. - Max. & $4.50 \pm 0.53$ & $4.75 \pm 0.46$ & $4.88 \pm 0.35$ & 2.706 & 0.258 \\
Mean \pm SD. & 4.50 & 5.0 & 5.0 & & \\
Median & &
\end{tabular}

H: H for Kruskal Wallis test, Pairwise comparison bet. each 2 groups was done using Post Hoc

Test (Dunn's for multiple comparisons test)

$P: P$ value for comparison between the three studied groups

$P_{1}: P$ value for comparison between control group and NAC

$P_{2}: P$ value for comparison between control group and CBL

$P_{3}: P$ value for comparison between NAC and CBL

*: Statistically significant at $P \leq 0.05$.

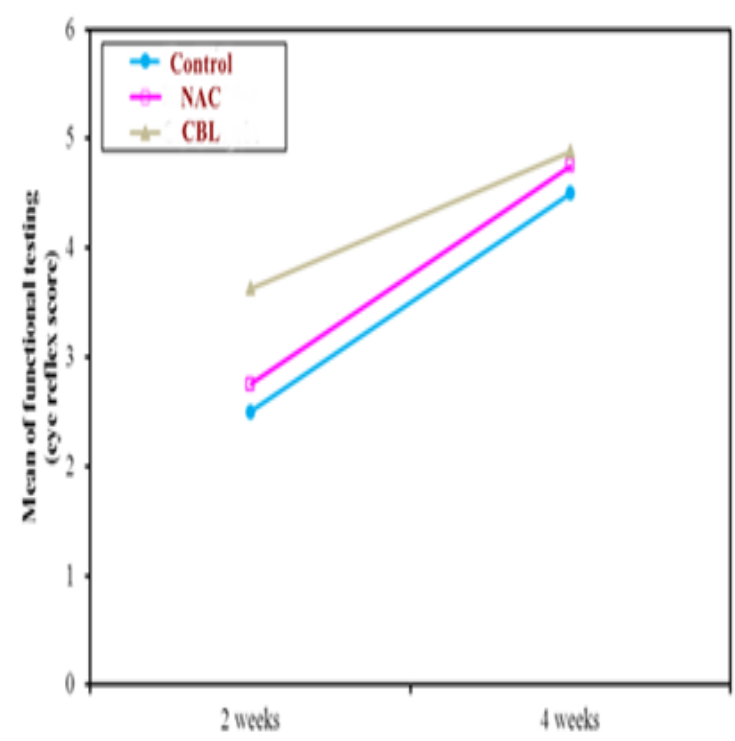

Figure 3: Comparison between the three groups according to functional testing (eye reflex score).

Wilcoxon test for comparison between the two periods according to functional testing representing statistically significant differences in control group $(\boldsymbol{P}=\mathbf{0 . 0 0 5})$, significant differences in NAC group $(\boldsymbol{P}=\mathbf{0 . 0 1 1})$ and significant differences in CBL group $(\boldsymbol{P}=\mathbf{0 . 0 0 8})$ as shown in (Table 2 and Figure 4).

Table 2: Comparison between the two periods according to functional test (eye reflex):

\begin{tabular}{lcccc}
\hline $\begin{array}{c}\text { Functional } \\
\text { testing } \\
\begin{array}{c}\text { (eye reflex } \\
\text { score) }\end{array}\end{array}$ & $\begin{array}{c}\text { 2weeks } \\
(\mathbf{n}=\mathbf{8})\end{array}$ & $\begin{array}{c}\text { 2weeks } \\
(\mathbf{n}=\mathbf{8})\end{array}$ & $\mathbf{Z}$ & $\boldsymbol{P}$ \\
\hline $\begin{array}{l}\text { Control } \\
\text { Median (Min. } \\
- \text { Max.) }\end{array}$ & $2.50(2.0-3.0)$ & $4.50(4.0-5.0)$ & & \\
$\begin{array}{l}\text { Mean } \pm \text { SD. } \\
\text { NAC }\end{array}$ & $2.50 \pm 0.53$ & $4.50 \pm 0.53$ & $2.828^{*}$ & $0.005^{*}$ \\
$\begin{array}{l}\text { Median (Min. } \\
- \text { Max.) }\end{array}$ & $3.0(2.0-4.0)$ & $5.0(4.0-5.0)$ & & \\
Mean \pm SD. & $2.75 \pm 0.71$ & $4.75 \pm 0.46$ & $2.549^{*}$ & $0.011^{*}$ \\
CBL & & & & \\
Median (Min. & $4.0(3.0-4.0)$ & $5.0(4.0-5.0)$ & & \\
- Max.) & $3.63 \pm 0.52$ & $4.88 \pm 0.35$ & 2.640 & $0.008^{*}$ \\
Mean \pm SD. & & & & \\
\hline
\end{tabular}

$\mathrm{Z}$ : Wilcoxon signed ranks test

$P$ : $P$ value for comparison between the two periods

*: Statistically significant at $P \leq 0.05$. 


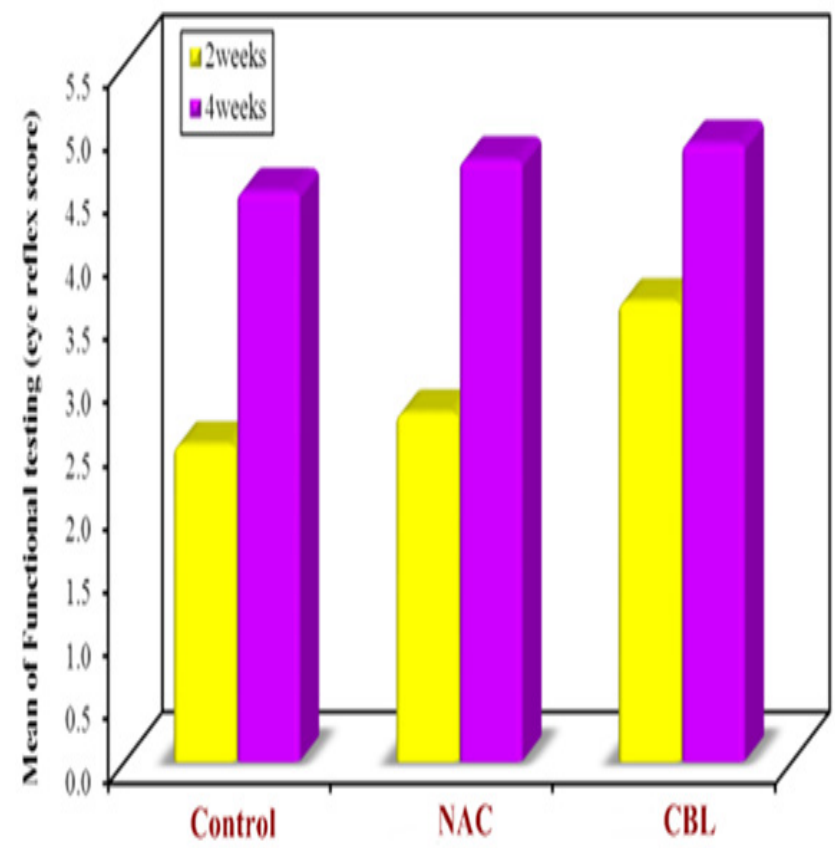

Figure 4: Comparison between the two periods according to functional testing (eye reflex score).

\section{B. Histopathological results (Hematoxylin and Eosin stain results):}

\section{Histopathological report:}

\section{Control group:}

The longitudinal examined sections of facial nerve in sacrificed control group rats revealed severe to intense histopathological lesions (Table 3) represented by numerous degenerated nerve bundles surrounded by extravasated erythrocytes (multifocal hemorrhages) among the local connective tissues and skeletal muscles bundles which admixed with inflammatory reactions as necrotic debris and inflammatory cells mainly lymphocytes. Moreover, the destructed and/or injured nerves suffered from demyelination, marked vacuolation axons besides disassociated axons as shown in (Figure 5).

Table 3: Facial nerve lesion score among different experimental groups:

\begin{tabular}{cccc}
\hline Lesion & Control & Cerebrolysin & $\begin{array}{c}\text { N-Acetyl } \\
\text { cysteine }\end{array}$ \\
\hline $\begin{array}{c}\text { Demyelination } \\
\text { of nerve axons }\end{array}$ & ++++ & - & ++ \\
$\begin{array}{c}\text { Perineural } \\
\text { hemorrhages } \\
\text { Neuronal }\end{array}$ & ++++ & + & ++ \\
$\begin{array}{c}\text { degeneration } \\
\text { / necrosis }\end{array}$ & ++++ & - & ++ \\
Vacuolation & ++ & + & +++ \\
$\begin{array}{c}\text { Congested } \\
\text { capillary }\end{array}$ & + & + & + \\
$\begin{array}{c}\text { Interstitial } \\
\text { cellularity }\end{array}$ & + & - & ++ \\
Regenerative \\
attempts
\end{tabular}

Pathological lesions Score system $(-=$ No Alterations $0 \%,+=$ Mild Alterations $10-35 \%,++=$ Moderate Alterations $40-50 \%,+++=$ Severe and $++++=$ Intense Alterations up to $60 \%$ ).
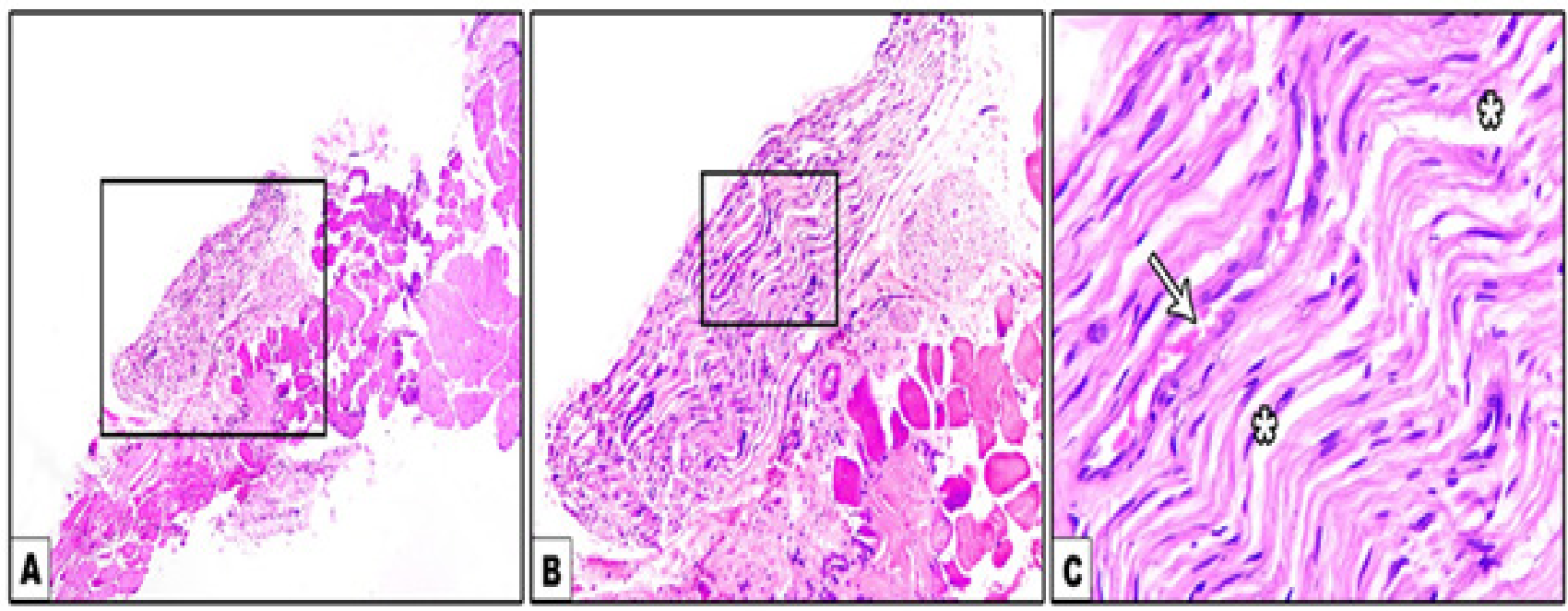

Figure 5: Representative photomicrograph of the rats ( $\mathrm{H}$ and $\mathrm{E}$ stained) facial nerve sections control group showing (A) numerous degenerated nerve branches surrounded by hemorrhages admixed with inflammatory process. X 40, (B and C) low and high magnification of injured facial nerve suffered from demyelination, marked separation (asterisks) with dilated blood vessel (arrow) X 100 and 400 respectively. 


\section{N-Acetyl cysteine group:}

In NAC treated group, there was mild to moderate regenerative attempts characterized by nearly normal facial nerve bundles surrounded by slightly focal hemorrhagic areas among the connective tissues and skeletal muscles. Moreover, other examined sections of wavy appearance of nerves suffered from mild to moderate vacuolated axons and slight congested capillary with normal perineurium and nodes of Ranvier (Figure 6).

\section{Cerebrolysin group:}

In CBL treated group, there was apparently normal facial nerve among connected tissues and masseter skeletal muscles with still presence of minute extravasated erythrocytes. The majority of nerve sections revealed restoration of the normal nerve structures as round or oval nuclei (Schwann cells) and elongated, densely stained nuclei (fibroblasts in the endoneurium) besides normal widening capillary with mild active endothelium followed by appeared normal perineurium as well as nodes of Ranvier as shown in (Figure 7).
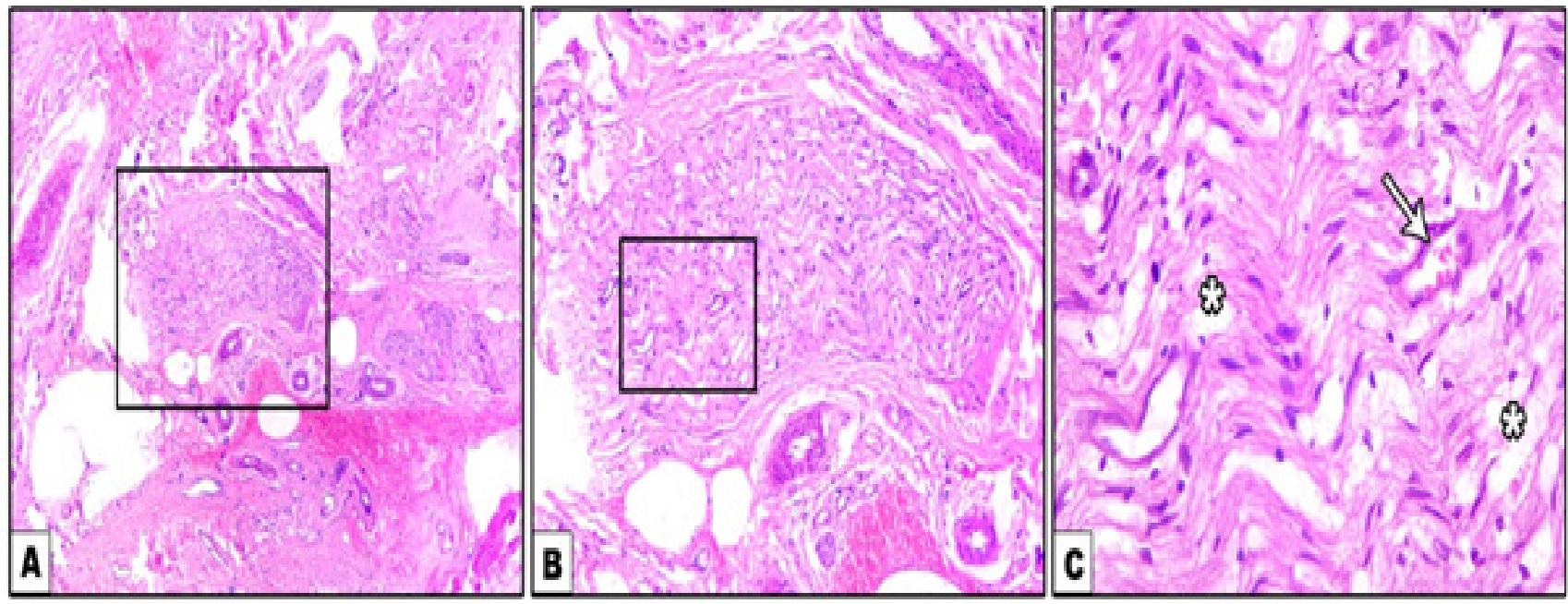

Figure 6: Representative photomicrograph of the rats $(\mathrm{H}$ and $\mathrm{E}$ stained) facial nerve sections treated with N-Acetyl cysteine group showing (A) mild degeneration of numerous nerve branches with presence of few extravasseted erythrocytes (rectangular) X 40. (B, C) low and high power of the rearranged facial nerve with segmentation intermingled with vacuolation (asterisks) with dilated blood vessel (arrow). X 100 and 400 respectively.
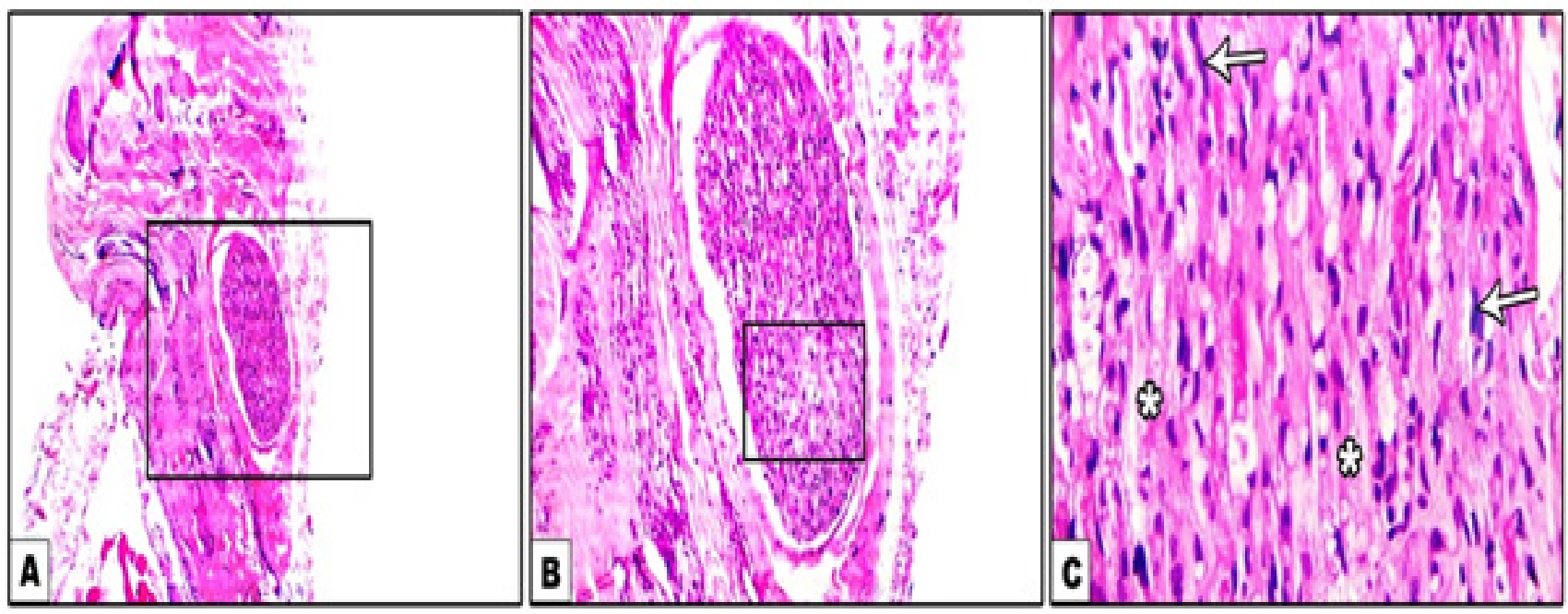

Figure 7: Representative photomicrograph of the rats (H and E stained) facial nerve section treated by CBL group showing (A) apparently normal nerve bundle (rectangular). X 40 (B and C) low and high magnification of the previous image to show remodeling of the nerve arrangement, elongated and densely stained nuclei (arrows) and remyelination (asterisks) without any histological alterations. X 100 and 400 respectively. 


\section{Immunohistochimechal stain:}

\section{Control group:}

$\mathrm{NF}-\kappa \mathrm{B}$ immunostaining was extensively noted in the longitudinal examined sections of facial nerve in sacrificed control group rats regard to inflammatory response. However, the immunoreactivity to S-100 protein showed mild staining expression. The expression of S-100 protein signal was located mainly in the myelin sheath as shown in (Figure 8).

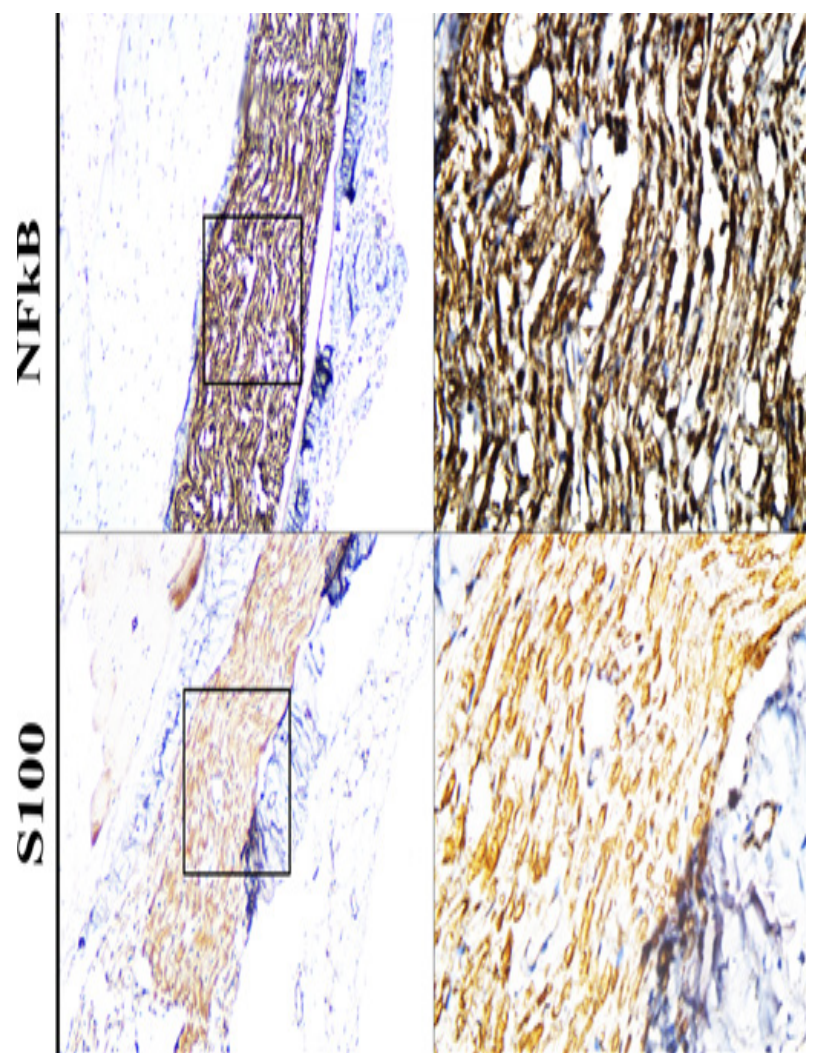

Figure 8: Representative photomicrograph of NFkB p65 and S100 IHC staining on the rat's facial nerve sections (X 100 and 400) control group showing severe brownish staining of NFkB expression in numerous nerve axons and SCs. While, mild intense brownish staining of S100 expression in majority of nerve axons and SCs were observed.

\section{N-Acetyl cysteine group:}

The longitudinal examined sections of facial nerve in sacrificed NAC treated group rats confirmed moderate to severe immunoreactivity to NF- $\kappa \mathrm{B}$.

Moreover, slightly regenerative attempts marked by mild to moderate intense of brownish color staining of S100 expression in main nerve axons and SCs as shown in (Figure 9).

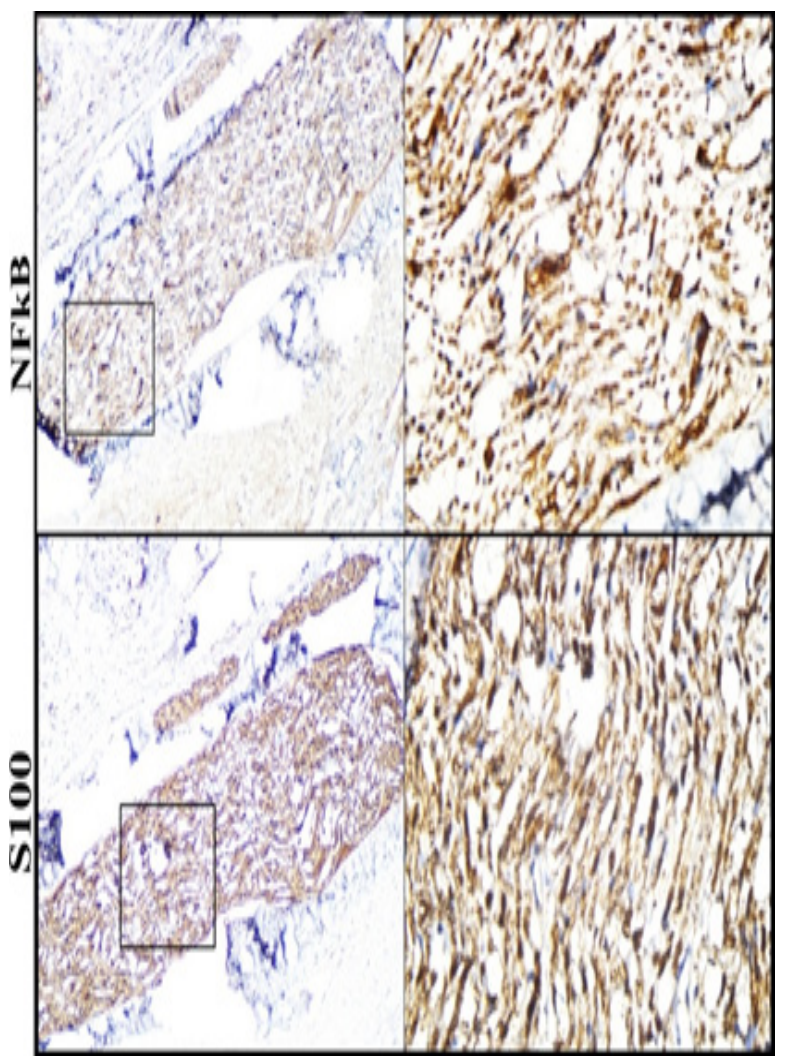

Figure 9: Representative photomicrograph of NFkB p65 and S100 IHC staining on the rat's facial nerve sections (X 100 and 400) NAC group showing moderate brownish staining of NFkB expression in a major nerve axons and SCs with still of them are intense. While, diffuse intense of brownish color staining of S100 expression in major nerve axons and SCs were common. 


\section{Cerebrolysin group:}

Immunoreactivity to NF- $\kappa \mathrm{B}$ showed mild staining in the longitudinal examined sections of facial nerve in sacrificed CBL treated group rats. While, the immunoreactivity to S-100 protein was extensively observed in the longitudinal sections of regenerated nerve segments. Expression of the S-100 protein was located mainly in the myelin sheath. There was distinctly more positive staining of the myelin sheath-associated protein S-100 as shown in (Figure 10).

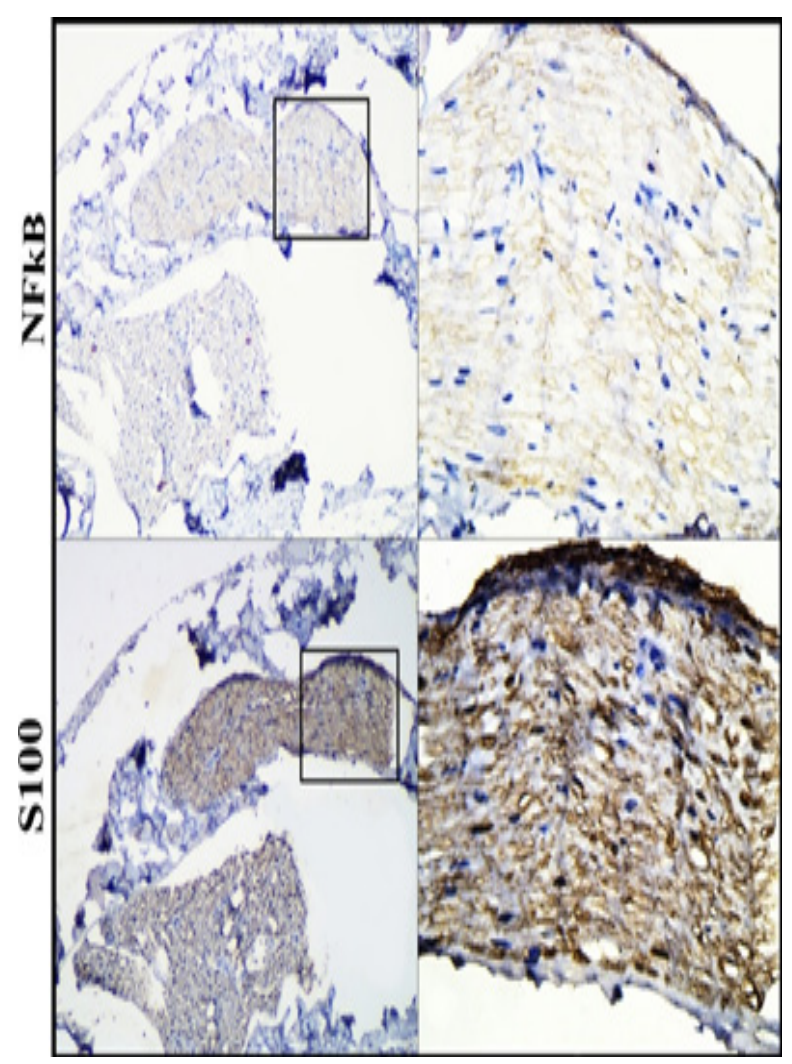

Figure 10: Representative photomicrograph of NFkB p65 and S100 IHC staining on the rat's facial nerve sections (X 100 and 400) CBL group showing very weak brownish staining of NFkB expression in a few nerve axons and SCs. While, markedly intense brownish staining of S100 expression in nerve axons, perineural sheath and SCs were noticed.

\section{Statistical analysis for immunohistochemical results:}

1) Comparison of Immunoreactivity to $S 100$ protein marker stain between all groups:

One-way ANOVA test for immunoreactivity to S100 represented statistical significant difference among all groups $(P<0.001)$. Post Hoc Test represented that control group was lower than NAC and CBL group regarding to immunoreactivity to $\mathrm{S} 100$ with statistical significant difference $\left(P_{1}=0.040\right),\left(P_{2}<0.001\right)$ respectively.
Additionally, the positive staining in myelin sheath with S100 marker stain in NAC group was lower than CBL group with statistical significant difference $\left(P_{3}<0.001\right)$, as shown in (Table 4 and Figure 11).

Table 4: Comparison among the three groups according to S100 IHC marker stain:

\begin{tabular}{cccccc}
\hline $\begin{array}{c}\text { S100 } \\
\text { IHC } \\
\text { marker } \\
\text { stain }\end{array}$ & $\begin{array}{c}\text { Control } \\
(\mathbf{n}=\mathbf{8})\end{array}$ & $\begin{array}{c}\text { NAC } \\
(\mathbf{n}=\mathbf{8})\end{array}$ & $\begin{array}{c}\mathbf{C B L} \\
(\mathbf{n}=\mathbf{8})\end{array}$ & $\mathbf{F}$ & $\boldsymbol{P}$ \\
\hline $\begin{array}{c}\text { Min. }- \\
\text { Max. }\end{array}$ & $120.9-176.0$ & $154.2-196.5$ & $200.5-267.1$ & & \\
$\begin{array}{c}\text { Mean } \\
\pm \text { SD. }\end{array}$ & $154.8 \pm 18.70$ & $179.3 \pm 16.99$ & $238.6 \pm 20.20$ & $42.614^{*}$ & $<0.001^{*}$ \\
& 159.3 & 189.2 & 245.8 & & \\
$\begin{array}{c}\text { Sig. } \\
\text { bet. } \\
\text { groups }\end{array}$ & $P_{1}=0.040^{*}, P_{2}<0.001^{*}, P_{3}<0.001^{*}$ & & \\
\hline
\end{tabular}

F: F for ANOVA test, Pairwise comparison bet. each 2 groups were done using Post Hoc Test

(Tukey)

$P: P$ value for comparison among the three groups

$P_{1}: P$ value for comparison between control group and NAC

$P_{2}: P$ value for comparison between control group and CBL

$P_{3}: P$ value for comparison between NAC and CBL

*: Statistically significant at $P<0.05$.

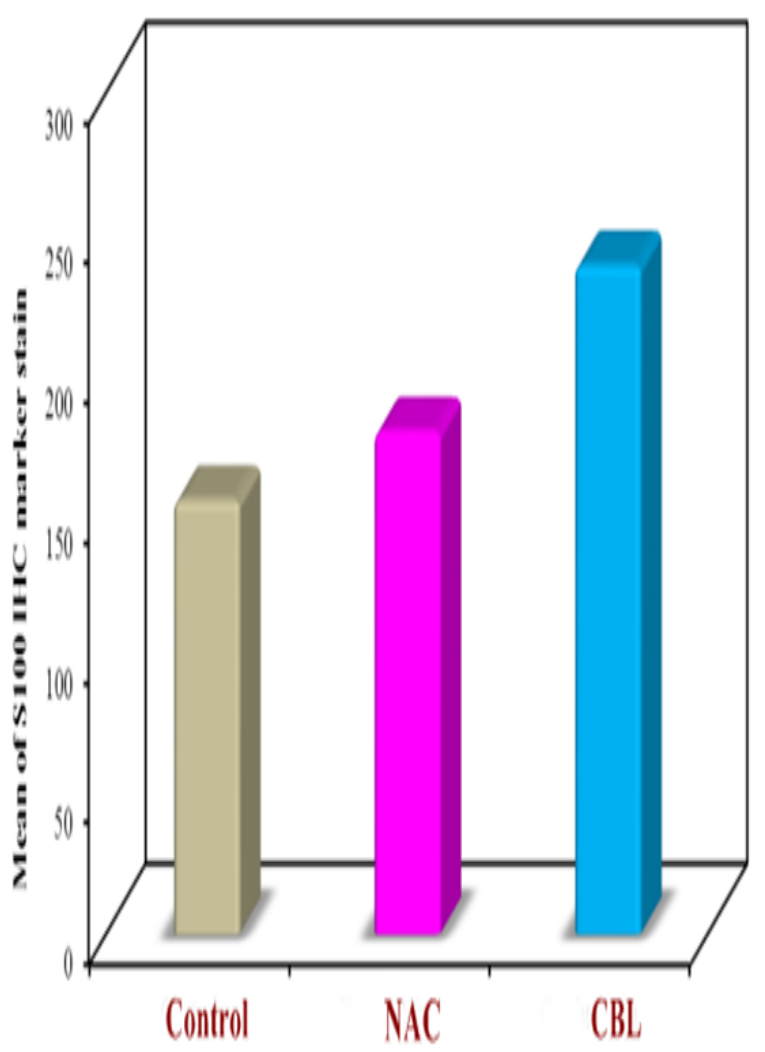

Figure 11: Comparison among the three groups according to S100 IHC marker stain. 


\section{2) Comparison of Immunoreactivity to NFkB marker stain between all groups:}

One-way ANOVA test for immunoreactivity to NFkB represented statistical significant difference among all groups $(P<0.001)$. Pairwise comparison between each 2 groups was represented that control group was higher than
NAC group and CBL group regarding to immunoreactivity to NFkB with statistical significant difference $\left(P_{1}=0.022\right),\left(P_{2}<0.001\right)$ respectively. Moreover, the positive staining to inflammatory response with NFkB marker stain in NAC group was higher than CBL group with statistical significant difference $\left(P_{3}<0.001\right)$, as shown in (Table 5 and Figure 12).

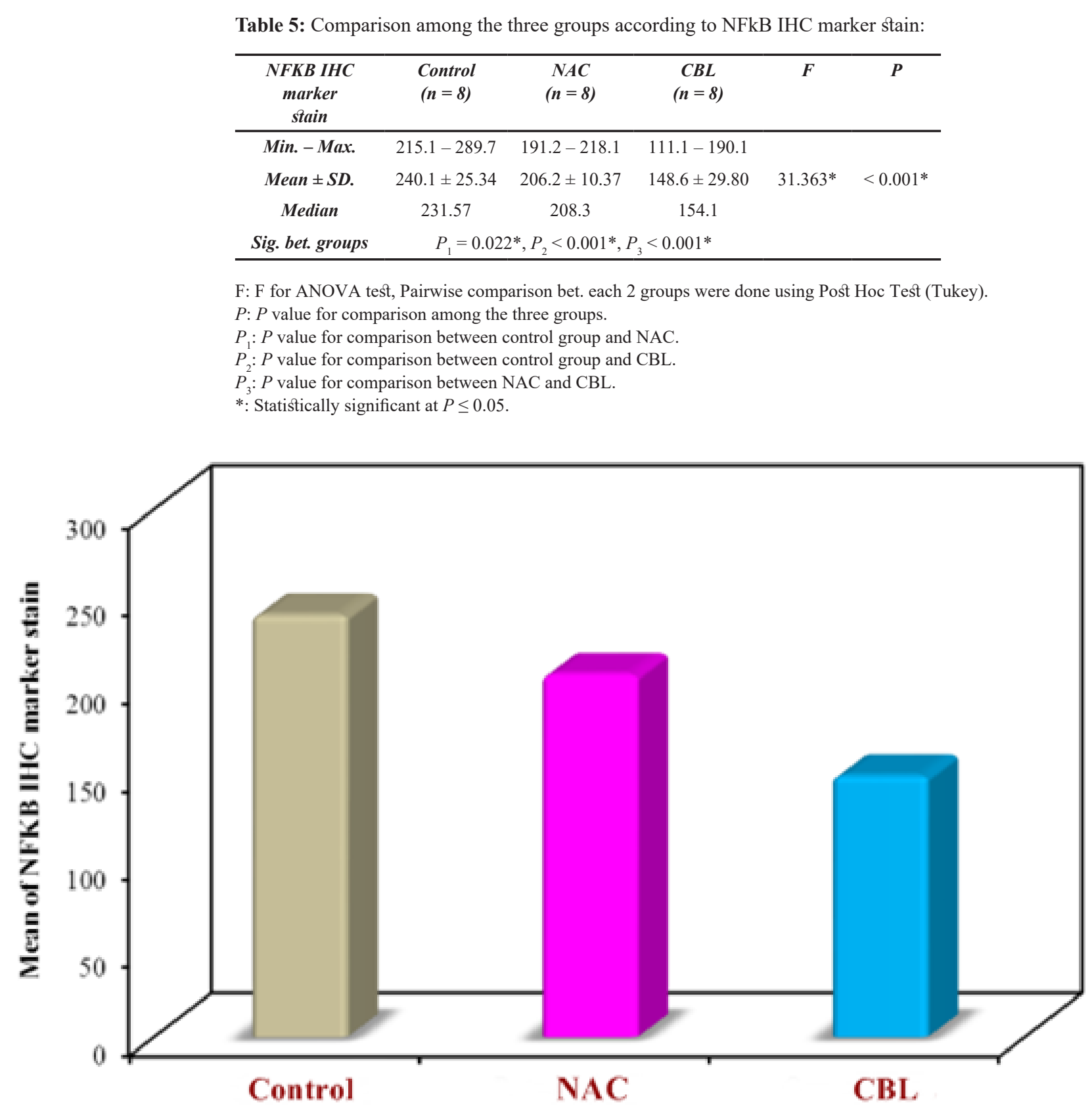

Figure 12: Comparison among the three groups according to NFkB IHC marker stain.

\section{DISCUSSION}

The facial nerve palsy is dramatic cranial nerve palsy with functional, esthetic and psychological problems that may occur when the symmetric structure between both sides of the face is disrupted due to $\mathrm{FN}$ lesions ${ }^{[19]}$. The management of facial paralysis includes surgical and nonsurgical options ${ }^{[20]}$.

Facial nerve dysfunction was treated previously by several medical therapies, whereas early steroids administration has shown improved functional outcomes 
in certain conditions of FN dysfunction, such as Bell's Palsy, our understanding is limited about the application of other neuroprotective agents in this setting ${ }^{[21,22]}$.

This study was conducted to evaluate the effectiveness of CBL and NAC on FN regeneration and rehabilitation after injuries and compare between them.

Different types of animals were used for the studies of nerve regeneration after injury. However, rats were used in this study, as Angius D, et al. showed that rats are economical, simple to handle and care for, very resistant to surgical infections, and can be investigated in large groups. It can be used for electrophysiology, functional recovery, muscle and nerve morphology and other assessments of nerve regeneration ${ }^{[23]}$.

The current study showed that administration of CBL intraperitoneally for up to 28 days in rats, significantly improved FN regeneration and rehabilitation in the experimentally induced traumatic facial paralysis model and after the nerve crushing, which is in agreement with the study of Hartbauer, et al. who demonstrated the neuro-protective function of CBL, and reported that $\mathrm{CBL}$ administration prevent the programmed cell death. They compared between control and CBL group regarding the lost cells as apoptosis contributes to a higher percentage of apoptotic neurons in the control, in that way scaling up the anti-apoptotic effect of $\mathrm{CBL}^{[24]}$.

Also, Chunling Zhang, et al. in their study agreed with this study and demonstrates that CBL dose improved neurological outcome. They stated that data show that CBL significantly augmented neurogenesis in the ischemic brain. Therefore, that neurogenesis increased may contribute to the improved functional outcomes ${ }^{[25]}$.

In agreement with this study results, Akai F, et al. observed that administration of CBL intraperitoneally for up to 4 weeks in rats after transection of fimbria-fornix in the brain, prevent the degeneration and atrophy of impaired septal cholinergic neurons ${ }^{[26]}$.

The current study revealed a significant change for the better in the functional outcomes of FN function after crush injury in those animals treated with CBL when compared with NAC or control group. Notably, statistical significance was demonstrated at the 2 weeks' postoperative assessment, indicating that systemic treatment with CBL might accelerate early FN recovery, probably by changing axonal sprouting or neuronal cell survival. Although there was no statistically significant difference demonstrated at the fourth postoperative week.

In agreement of these results, Hamed SH, demonstrated the therapeutic efficacy of CBL in the treatment of acquired peripheral nerve system diseases. The improved neuronal recovery may result from decreased excitotoxic damage and increased nerve viability and survival after trauma or inflammation ${ }^{[27]}$.
While, in contrast to this study, Andrew Rivera et al. who compared between NAC group and control group on FN regeneration, revealed that NAC was demonstrated a significant increase in nerve regeneration at nerve transection model, also they stated that there was statistically significant in eye blinking function at 4 weeks with NAC group ${ }^{[12]}$.

This study revealed that NAC had slight effect on FN regeneration and rehabilitation; and remyelinization was significantly lower in NAC administered group when compared to CBL treated group.

Also, contradictively, Turgut Karlidag, et al. in their study compared between NAC and methylprednisolone; and they observed that NAC administration caused significant improvement in the nerve regeneration after FN traumatic injury ${ }^{[28]}$.

While in group II of this study which treated with NAC, there were insignificant regenerative attempts in FN model after crush injury and administration of NAC, when compared with CBL group.

Results of this study revealed that facial nerve regeneration was better in the group who received CBL administration, the data revealed that $\mathrm{CBL}$ therapy improved histological and functional outcomes from FN crush injury in rats without causing an increase in fibrotic tissue following the injury, perhaps by improving the regional blood supply and balancing the anti-inflammatory and antioxidant effects.

In agreement with these results, Nasser Zangiabadi, et al. mentioned that microscopic investigations showed significant improvement in myelin and axon diameter reduction among diabetic rats in two weeks with group which treated by CBL administration ${ }^{[29]}$.

Immunohistochemical staining for $\mathrm{NF}-\kappa \mathrm{B}$ and $\mathrm{S} 100$ proteins were performed with $\mathrm{H}$ and $\mathrm{E}$ in this study to confirm results as Vleggeert-Lankamp stated that histomorphometry has been the most common and frequently used outcome measure evaluating regeneration of peripheral nerve and it persists the most commonly used valuation at present time ${ }^{[29]}$.

The myelin sheath markers (S100 proteins) are expressed in peripheral neurons and normally present in cells derived from the neural crest (SCs, and melanocytes). So, a successful IHC analysis, that may be used to assess the morphological structure of peripheral neurons, is in need of the presence of S100 proteins ${ }^{[30]}$.

Mekaj, et al. mentioned that $\mathrm{S} 100$ protein expression was primarily located in the myelin sheath, in the longitudinal sections, of the regenerated nerve segments and the expression was as intensive cytoplasmic staining ${ }^{[30]}$.

Immunohistochemical staining revealed improved regeneration and positive staining of the myelin sheath associated protein S-100 in CBL group more than NAC group after FN injury. Therefore, $\mathrm{CBL}$ was able to enhance 
several of the primary SCs functions following peripheral nerve injury, indicating that $\mathrm{CBL}$ may be an effective therapeutic agent. In this study, a protein complex NF-кB (Nuclear factor kappa-B) was used, which acts on genes for pro-inflammatory cytokines, chemokine's (chemotactic cytokines that attract inflammatory cells to inflammation sites), immune receptors, enzymes that generates inflammation mediators, and adhesion molecules that play a key role in the initial recruitment of leukocytes to sites of inflammation. The activation of NFkB therefore leads to a coordinated increase in the expression of many genes whose acts a key part in regulating the immune response to inflammation $^{[31]}$.

Results of this study showed that mild staining was observed in axons and SCs associated NF- $\kappa \mathrm{B}$ immunostaining in $\mathrm{CBL}$ group which revealed that $\mathrm{CBL}$ reducing the effect of crush injury may be related with anti-inflammatory properties of CBL. In agreement with these study results, Kizilay, et al. observed that NF-Kb immunoreactivity was weaker in their treatment group and NF- $\kappa \mathrm{B}$ is a redox sensitive transcriptional factor and has been reported to be activated via oxidative stress, hyperglycemia and pro-inflammatory cytokines ${ }^{[32]}$. Toader, et al. also agreed with the current study and observed in their immunohistochemistry study that CBL decreased inflammation and alleviated some of the pathological denominators like demyelination, neuronal cytoskeleton loss and microglia density. A moderate protection also appeared at the level of the optic nerve, where showed a degree of demyelination, compared to control $\operatorname{animals}^{[33]}$.

\section{CONCLUSION}

It can be accomplished that CBL enhances facial nerve regeneration and rehabilitation after nerve crush injury. Also, it is an effective preventive drug that reduces inflammation. According to these effects $\mathrm{CBL}$ is a superior drug when compared with NAC.

\section{CONFLICT OF INTEREST}

The authors declare no conflict of interest.

\section{REFERENCES}

1. Gordin E, Lee T, Ducic Y, Arnaoutakis D. Facial Nerve Trauma: Evaluation and Considerations in Management. Craniomaxillofacial Trauma Reconstr 2015; 8: 1 - 13 .

2. Gupta S, Mends F, Hagiwara M, Fatterpekar $\mathrm{G}$, Roehm PC. Imaging the facial nerve: a contemporary review. Radiol Res Pract 2013; 2: $1-14$.
3. Bayrak A, Olgun Y, Ozbakan A, Aktas S, Kulan C, Kamaci, G Olgun L. The Effect of Insulin Like Growth Factor-1 on Recovery of Facial Nerve Crush Injury. Clin Exp Otorhinolaryngol 2017; 10: $296-302$.

4. Seddon HJ. A Classification of Nerve Injuries. Br Med J 1942; 2: 237 - 9.

5. May M, Schaitkin, Barry M. The Facial Nerve. 2000; 83: $179-95$.

6. Lee LN, Lyford-Pike S, Boahene KDO. Traumatic Facial Nerve Injury. Otolaryngol Clin North Am 2013; 46: $825-39$.

7. Dölen T, Kaygusuz İ, Akpolat N, Alpay HC, Karlıdağ T, Yüksel K. The effect of nimodipine and prednisolone on traumatic facial nerve injury treatment. ENT Updat 2017; 7: $1-8$.

8. Ozturk O, Tezcan AH, Adali Y, Yıldırım $\mathrm{CH}$, Aksoy $\mathrm{O}$, Bilge A. Effect of ozone and methylprednisolone treatment following crush type sciatic nerve injury. Acta Cir Bras 2016; $31: 730-5$.

9. Deepmala, Slattery J, Kumar N, Delhey L, Dean $\mathrm{O}$, Frye R. Clinical trials of N-acetylcysteine in psychiatry and neurology: A systematic review. Neurosci Biobehav Rev 2015; 55: 294 - 321.

10. Karalija A, Novikova LN, Kingham PJ, Wiberg M, Novikov LN. The effects of N-acetylcysteine and acetyl-1-carnitine on neural survival, neuroinflammation and regeneration following spinal cord injury. Neuroscience 2014; 269: $143-51$.

11. Plosker GL, Gauthier S. Cerebrolysin, A Review of its Use in Dementia. Drugs and Aging 2009; 26: $893-915$.

12. Rivera A, Raymond M, Grobman A, Abouyared M, Angeli S. The Effect of N-Acetyl-Cysteine on Recovery of the Facial Nerve After Crush Injury. Laryngoscope Investig Otolaryngol 2017; 272: $473-8$.

13. Borin A, Toledo RN. Standardization of techniques used in facial nerve section and facial movement evaluation in rats. Rev Bras Otorrinolaringo 2006; 72: $341-7$.

14. Olmstead DN, Mesnard-Hoaglin NA, Batka RJ, Haulcomb MM, Miller WM, Jones KJ. 
Facial Nerve Axotomy in Mice: A Model to Study Motoneuron Response to Injury. J Vis Exp 2015; 96: $1-7$.

15. Matsumine H, Sasaki R, Takeuchi Y, Watanabe Y, Niimi Y, Yamato M. Unilateral multiple facial nerve branch reconstruction using 'end-to side loop graft' supercharged by hypoglossal nerve. Plast Reconstr Surg - Glob Open 2014; 2: 1 - 11 .

16. Stepanichev M, Onufriev M, Aniol V, Freiman $\mathrm{S}$, Brandstaetter H, Gulyaeva N. Effects of cerebrolysin on nerve growth factor system in the aging rat brain. Restor Neurol Neurosci 2017; 35: $571-81$.

17. De Faria SD, Testa JR, Borin A, Toledo RN. Standardization of techniques used in facial nerve section and facial movement evaluation in rats. Brazilian Journal of Otorhinolaryngology. 2006; $72: 341-7$.

18. Suvarna KS, Layton C, Bancroft JD. Bancroft's theory and practice of histological techniques. In: Eighth edition. Elsevier Health Sciences, $2018,40-63$.

19. Tunçcan T, Yalçın Ş, Demir CF, Akın MM, Karlıdağ T, Kaygusuz İ. Efficacy of Cortexin and Methylprednisolone on Traumatic Facial Nerve Paralysis. J Int Adv Otol 2016; 12: 5 - 9.

20. Owusu JA, Stewart CM. Facial Nerve Paralysis. Med Clin NA 2018; 102: 1135 - 43.

21. Sullivan FM, Swan IR, Donnan PT, Morrison JM, Smith BH, McKinstry B, Davenport RJ, Vale LD, Clarkson JE, Hammersley V, Hayavi S, McAteer A, Stewart K, Daly F. Early treatment with prednisolone or acyclovir in Bell's palsy. N Engl J Med. 2007; 357: 1598 - 607.

22. Engstrom M, Berg $\mathrm{T}$, Stjernquist-Desatnik A, Axelsson S, Pitkaranta A, Hultcrantz M, Kanerva M, Hanner P, Jonsson L. Prednisolone and valaciclovir in Bell's palsy: a randomized, doubleblind, placebo-controlled, multicentre trial. Lancet Neurol. 2008; 7: 993 - 1000.

23. Angius D, Wang H, Spinner R, Cotto Y, Yaszemski M, Windebank A.A systematic review of animal models used to study nerve regeneration in tissue engineered scaffolds. NIH Public Access 2013; 33: $8034-9$.

24. Hartbauer M, Hutter-Paier B, Skofitsch G, Windisch M. Antiapoptotic effects of the peptidergic drug Cerebrolysin on primary cultures of embryonic chick cortical neurons. J Neural Transm 2001; 108: $459-73$.

25. Zhang C, Chopp M, Cui Y, Wang L, Zhang R, Hitzl M. Cerebrolysin Enhances Neurogenesis in the Ischemic Brain and Improves Functional Outcome After Stroke. J Neurosci Res 2010; 88: $3275-81$.

26. Akai F, Hiruma S, Sato T, Iwamoto N, Fujimoto M, Hashimoto S. Neurotrophic factor-like effect of FPF1070 on septal cholinergic neurons after transections of fimbria-fornix in the rat brain. Histol Histopathol 1992; 7: 213 - 21.

27. Hamed SA. Cerebrolysin as a nerve growth factor for treatment of acquired peripheral nervous system diseases. NEURAL Regen Res $2011 ; 61415-20$.

28. Karlidag T, Yildiz M, Yalcin S, Colakoglu N, Kaygusuz I, Sapmaz E. Evaluation of the effect of methylprednisolone and $\mathrm{N}$-acetylcystein on anastomotic degeneration and regeneraton of the facial nerve. Auris Nasus Larynx 2012; 39: $145-50$.

29. Zangiabadi N, Mohtashami H, Shabani M, Jafari M. Neuroprotective Effect of Cerebrolysin on Diabetic Neuropathy: A Study on Male Rats. Diabetes Metab 2014; 5: $1-7$.

30. Mekaj, A. Y. Mekaj, S. Manxhuka Kerliu, A. A. Morina, S. B. Duci, L. Shahini YH. Effects of hyaluronic acid and tacrolimus on the prevention of perineural scar formation and on nerve regeneration after sciatic nerve repair in a rabbit model. Eur $\mathrm{J}$ Trauma Emerg Surg 2017; 43: $497-504$.

31. Barnes P, Karin M. Nuclear Factor-k B- A Pivotal Transcription Factor in Chronic Inflammatory Diseases. N Engl J Med 1997; 336: 1066 - 71.

32. Kızılay Z, Çetin NK, Aksel M, Abas Bİ, Akta S. Ozone Partially Decreases Axonal and Myelin Damage in an Experimental Sciatic Nerve Injury Model. J Investig Surg 2017; 1939: 1 - 10.

33. Toader, Emilia L, Roşu G-C, Cătălin B, Fior D, Pirici. D. Cerebrolysin increases motor recovery and decreases inflammation in a mouse model of autoimmune encephalitis. Rom J Morphol Embryol 2018; 59: 755 - 62 . 